\title{
Searching the Visual Style and Structure of D3 Visualizations
}

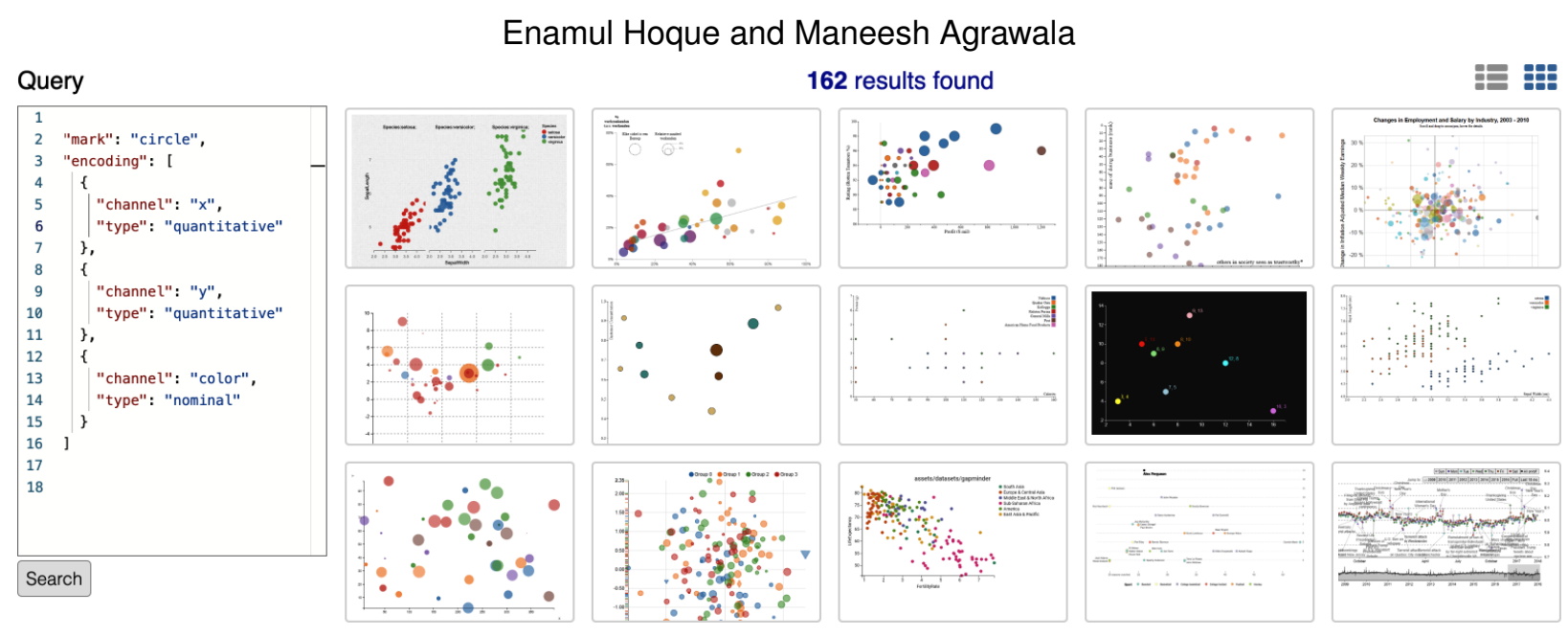

Fig. 1. Our search interface lets users query for D3 visualizations based on the data, the marks and the encodings that describe how visual attributes of the marks represent the data. Our query syntax is based on the Vega-Lite chart specification grammar [40]. Here, the user searches for visualizations that use circles as data-encoding marks and contain at least three encodings. The query specifies that two of the encodings must map quantitative data fields to the x-position and the y-position of the circle marks respectively, while a third encoding must map a nominal data field to the fill-color of the marks. The query results include a variety of scatterplot and bubble chart styles that can help developers explore the design space of such visualizations.

Abstract-We present a search engine for D3 visualizations that allows queries based on their visual style and underlying structure. To build the engine we crawl a collection of 7860 D3 visualizations from the Web and deconstruct each one to recover its data, its data-encoding marks and the encodings describing how the data is mapped to visual attributes of the marks. We also extract axes and other non-data-encoding attributes of marks (e.g., typeface, background color). Our search engine indexes this style and structure information as well as metadata about the webpage containing the chart. We show how visualization developers can search the collection to find visualizations that exhibit specific design characteristics and thereby explore the space of possible designs. We also demonstrate how researchers can use the search engine to identify commonly used visual design patterns and we perform such a demographic design analysis across our collection of D3 charts. A user study reveals that visualization developers found our style and structure based search engine to be significantly more useful and satisfying for finding different designs of D3 charts, than a baseline search engine that only allows keyword search over the webpage containing a chart.

Index Terms-visualization search engine; visualization design; search user interfaces

\section{INTRODUCTION}

The popularity of Web-based data visualizations has grown rapidly, as people frequently use them to explore datasets and present complex ideas. Many of these visualizations are created using D3.js, a JavaScript library [6], with a large community of developers. Sites like the New York Times [13], the Wall Street Journal [19], Bloomberg [4] and Pudding.cool [17] regularly publish D3 visualizations to tell stories with data. Thousands of D3 charts are available online, depicting many different datasets and exhibiting a wide variety of visual designs.

The large collection of D3 visualizations available online serves as a resource for visualization developers and researchers to explore the space of visualization designs, and to analyze design demographics. For instance, visualization developers often seek example charts to re-use or adapt the D3 code rather than writing the code from scratch. Such developers also use online examples to better understand design choices

\footnotetext{
- Enamul Hoque is with York University. E-mail: enamulh@yorku.ca.

- Maneesh Agrawala is with Stanford University. E-mail: maneesh@cs.stanford.edu.
}

Manuscript received xx xxx. 201x; accepted xx xxx. 201x. Date of Publication xx xxx. 201x; date of current version xx xxx. 201x. For information on obtaining reprints of this article, please send e-mail to: reprints@ieee.org. Digital Object Identifier: $x x . x x x x / T V C G .201 x . x x x x x x x$ (e.g. encoding data using size vs. color) and stylistic decisions (e.g. typefaces, color palettes). Researchers may be interested in analyzing the most frequently used design patterns (e.g. which visual attribute - position, length or color - is most commonly used to encode data?) or how often designers violate best practices (e.g. limit the number of colors used to encode nominal data to between six and twelve [34 45]). Such design patterns can provide a better understanding of real-world design practices.

Today such design exploration and demographic analysis of Webbased D3 visualizations is difficult. Often the first step is to search for a representative set of D3 charts. But, generic search engines (e.g. Google, Bing) index the entire Web and do not provide any way to search only the pages containing D3 charts. In contrast, the Sightline [42] search engine and manually curated D3 collections [23] only include webpages containing D3 charts. While these tools make it easier for visualization developers to find D3 charts, none of them provide access to the visual style, structure and content of the charts. Therefore, it is impossible for example to query for the set of D3 charts that encode data using rectangle height, or to retrieve all of the charts that use a dark background. Instead developers and researchers must look at each D3 chart in the collection and manually check whether its visual design satisfies the query.

In this paper, we present a search engine that indexes D3 visualizations based on their visual style and structure to directly support such 


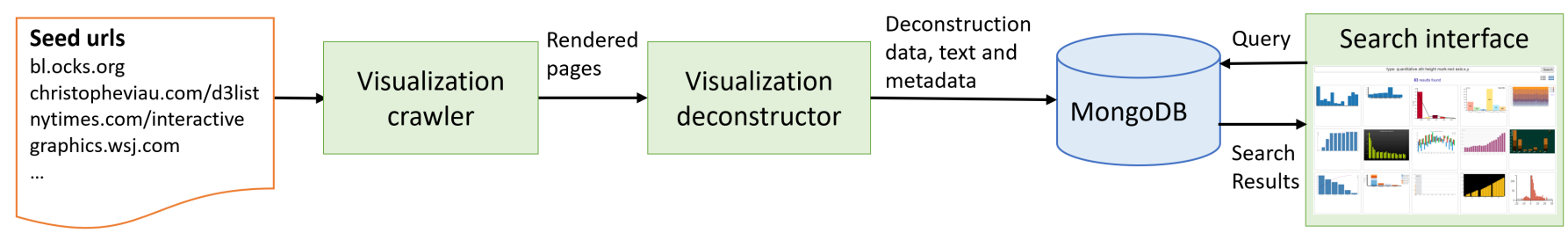

Fig. 2. Our visualization search engine is comprised of three main components (green boxes): a visualization crawler, a visualization deconstructor and a search interface. The crawler finds webpages containing D3 charts, the deconstructor extracts the visual style and structure of the charts (e.g. the data, the marks and the encodings) and the search interface lets users query for charts that include specific aspects of style and structure.

design queries. To build the engine we crawl a collection of $7860 \mathrm{D} 3$ charts from the Web and extend Harper and Agrawala's D3 Deconstructor [26 27], to recover their constituent data, data-encoding marks (e.g. rectangles in bar chart, circles in a scatterplot) and the encodings describing how the data is mapped to visual attributes of the marks (e.g. area, height, fill-color). We also extract the visual attributes of axes (e.g. attributes of tick marks and axis labels) and other non-data-encoding attributes of marks (e.g. typeface, background color) as these provide additional information about the style of the visualization. Our search engine indexes this style and structure information as well as the text and metadata of the webpage containing each chart.

Our query syntax is based on the Vega-Lite [40]/CompassQL [47] chart specification grammar that declaratively represents a chart as a collection of encodings that map data to visual attributes of marks. For example, as shown in Figure 1, users can search for all charts using circle marks in which the x-position and y-position are mapped to quantitative data fields while fill-color is mapped to a nominal data field. This search yields a set of scatterplots and bubble charts that contain at least these three encodings. We show how such search results can help visualization developers explore the variety of charts that can be produced for a given set of encodings or design characteristics. We also demonstrate how researchers can use our search engine to identify common visual design patterns and we perform such a demographic design analysis across our collection of charts. A user study comparing our search interface which indexes the style and structure of D3 charts, with a baseline interface (Sightline [42]) that only provides keyword search over the webpage containing a D3 chart, reveals that visualization developers find our interface significantly more useful and satisfying to use as it allows them to quickly find different designs and styles of D3 charts.

\section{Related Work}

Deconstructing visualizations: Constructing a chart involves encoding the data by mapping it to some visual attributes (e.g. position, color) of marks (e.g. circles, rectangles). Deconstructing charts is the inverse process - i.e., given a chart, the goal is to extract the underlying data, the marks with their visual attributes, and mappings (or encodings) between them. There has been growing interest in automatically deconstructing visualizations from bitmap images of charts. ReVision [41] identifies chart type using an SVM-based classifier then extracts the underlying marks and data from an input chart image. ChartSense [29] uses a convolutional neural network $(\mathrm{CNN})$ for chart type classification and a mixed-initiative approach to extract the marks and data. Others have focused on extracting text from chart images (e.g., axis labels) [36], recovering color encodings by by analyzing the legend of a chart [37] and recovering data from scatterplots [24]. These techniques are orthogonal to our work as they are designed to recover data, marks and encodings from bitmaps of charts, while our goal is to index visualizations based on the deconstructed data, marks and encodings. As these methods improve in accuracy we believe it should be possible to use them in conjunction with our methods to index the visual style and structure of bitmap charts.

More recently, Harper and Agrawala [26, 27] have developed techniques for deconstructing D3 visualizations. Because they work from a programmatic description of the chart they can extract the data, the marks and the encodings far more accurately than bitmap-based techniques. Our work extends their deconstruction approach to extract additional features including non data-encoding marks and their visual attributes. Our search engine indexes this information and our query syntax enables search over a broad range of the chart design space, covering both style and structure.

Indexing and searching visualizations: Several researchers have developed techniques for indexing, and searching Web-based visualizations. For example, Sightline [42] is a chrome extension that automatically indexes data visualizations encountered by users. It then supports retrieval based on keyword search over the text and metadata (e.g. author, title) of the webpage containing the visualization. Graphical Histories [28] and KnowledgePearls [44] are visual analysis tools that record user interactions and the state of the visualization after each step of the analysis in a provenance graph. Users can then query for previous exploration states. Others have developed techniques for searching for charts in a collection of documents based on their text labels (e.g., axes labels, titles, legends, and captions) [23,36, 43]. Lee et al. [31] extract images from a large corpus of scientific papers and classify the figure types (e.g., equation, diagram, plot and table). Unlike these techniques our search engine indexes lower-level aspects of visual style and structure, allowing search by data, mark type and encoding.

Analyzing design demographics: Our work is inspired by recent efforts on mining large corpora of Web documents to facilitate demographic analysis in various domains such as webpage design [30 38] and API usage [25]. However, analysis of visualization design demographics has been rare. Beagle [21] classifies several thousands SVG-based data visualizations by chart type (e.g. bar chart, scatterplot). They find that the majority of charts in the collection only cover four types: bar charts, line charts, scatterplots, and geographic maps. We conduct a broader demographic analysis of visualization design covering the usage of different mark types and encodings.

\section{Search Engine for D3 Visualization}

As shown in Figure 2, our search engine consists of three major components: (1) The visualization crawler downloads webpages starting from a collection of seed URLs. (2) The visualization deconstructor extracts the visual style and structure of the D3 visualizations found on each crawled webpage and stores the deconstructed information in a database. (3) The search interface then lets end-users query the database based on the style and structure information.

\subsection{Visualization Crawler}

Our visualization crawler extracts weblinks from a set of seed URLs, then downloads the corresponding webpage and finally renders the resulting page in the Chrome browser so that we can apply our visualization deconstructor to the page. Our crawler only follows one level of links, and we seed it with webpages containing lists of links to D3 visualizations. Members of the D3 developer community have compiled several such lists at sites like d3.js [6], bl.ocks.org [3] and the "Big List of D3 Examples" [2]. We supplement these community curated seed lists with additional lists of interactive visualizations that are available directly from news sites like the New York Times [14] and the Wall Street Journal [19].

In total we crawled 2623 webpages from 622 different domains, where each page contained at least one D3 visualization, and we deconstructed a total of 7860 D3 visualizations. We also captured screenshots of the visualization as well as the HTML of the webpage it was embedded in. The resulting database takes 5.6 GB of disk storage. Supplemental Materials: Figure 1 shows the top 20 domains in terms of the number of D3 visualizations we extracted from them. 
(C) 2019 IEEE. This is the author's version of the article that has been published in IEEE Transactions on Visualization and Computer Graphics. The final version of this record is available at: 10.1109/TVCG.2019.2934431

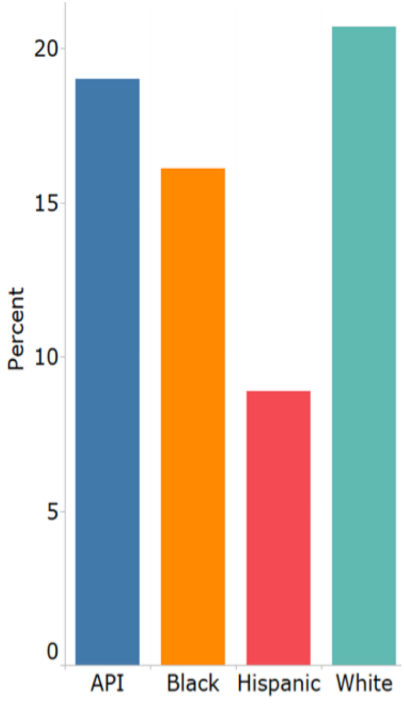

a) Bar chart

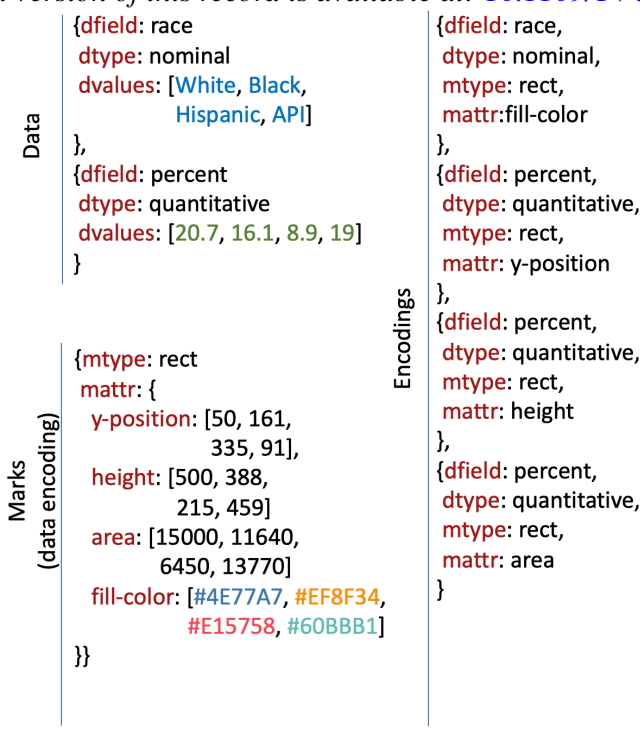

b) Deconstruction

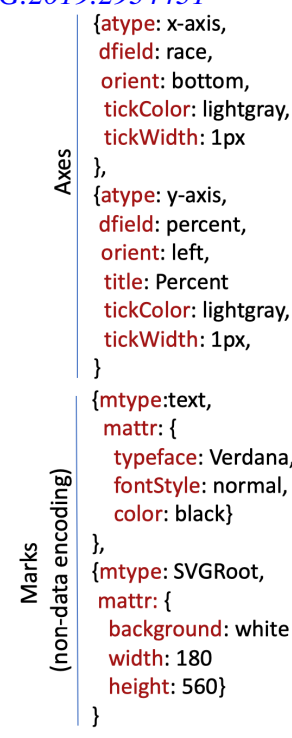



\section{c) Vega-Lite}

Fig. 3. A D3 bar chart (a). The data, marks, encodings, axes and non-data-encoding attributes of marks as extracted by our deconstructor (b). In this case, our deconstructor extracts two data fields named "race" and "percent" of data types "nominal" and "quantitative" respectively. It also extracts data-encoding marks of type "rect" and a set of 4 encodings that map data fields to visual attributes of the rect marks (e.g., the data field "race" is mapped to the fill-color of "rect"). Finally, it extracts the axes, including the data field they represent and their orientation and non-data-encoding attributes of the marks (e.g. typeface, background color). A Vega-Lite specification for the bar chart is similar to our deconstructed representation and explicitly describes the relevant encodings (c).

\subsection{Visualization Deconstructor}

The goal of our visualization deconstructor is to extract the complete visual style and structure of every D3 chart that appears on an input webpage (Figure 3). Each data field in our deconstruction is is represented by a field name ' 'dfield' ', data type ' 'dtype', , and a set of data values ' "dvalues' '. In Figure 3 one data field is named race with data type nominal, while the other data field is named percent with data type quantitative. The data encoding marks are represented by their mark types ' 'mtype' ' which can take values like rect, circle, line, path, and text as well as mark attributes ' 'mattr' ' which can take values like fill-color, opacity, size, angle, x-position and y-position. Each data-encoding mark attribute can also take a set of values. For example $y$-position has four values each of which corresponds to the $y$-position of a rect mark in screen space. Our deconstructor analyzes the relationships between data values and the values of each mark attribute to recover the encodings that map between them - represented as $\{$ dfield, dtype, mtype, mattr $\}$. For example, the encoding at the top of Figure $3 \mathrm{~b}$ (middle column) specifies that the race data field is mapped to the fill-color of the data-encoding rect marks.

Our deconstructor also extracts each axis, including the axis type " 'atype', either $x$-axis or $y$-axis, the data field "dfield" it represents, its orientation ' 'orient' ' either left, right, bottom or top and other axis attributes such as " title',' ' 'tickColor', ' 'tickWidth', etc. Finally it extracts non-data-encoding attributes of the marks such as typeface and background color. Note that our deconstructor treats the root SVG node of the D3 chart a non-dataencoding mark and extracts its attributes as well.

Our deconstructor builds on Harper and Agrawala's D3 Deconstructor [26 27] which focused on recovering the data, the marks and the encodings from a D3 chart. We extend their deconstructor in three key ways: (1) We extract an additional visual attribute angle for marks instantiated using D3's d3. $\operatorname{arc}()$. Such arcs are typically used to create pie charts, donut charts and radial bar charts which encode data using the angle of the arc. (2) We extract visual attributes that do not typically encode data but are used instead for styling (e.g., background color, typeface, stroke-width) from all the marks and SVG elements including text elements and the top-level SVG node. (3) We extract axes and their attributes such as the data fields they represent, their orientation, their title text, etc. The resulting deconstruction is very similar to a Vega-Lite specification which focuses on the encodings between data and mark attributes (Figure 36) We describe these exten- sions in detail in the supplemental material. We store the deconstruction data in a MongoDB database [12]. We chose MongoDB since it is suitable for processing the hierarchical structure in JSON format that our deconstructor generates.

\subsection{Search User Interface}

Our search interface lets users query the database of deconstructed D3 visualizations by providing a partial specification of the desired results. We first describe our query syntax and then present the interface features for exploring the search results.

\subsubsection{Query syntax}

Our query syntax is based on the Vega-Lite [40] grammar for declaratively specifying a chart as a collection of encodings that map data to visual attributes of marks (Figure 3.). We chose the Vega-Lite syntax because it explicitly describes the structure and style of a visualization and is similar to other grammar-based representations [7, 46]. Grammar-based specifications like Vega-Lite have proven to be an effective representation for conceptualizing visualizations [46] because they help developers understand the way charts are constructed from data, marks and encoding. Moreover, Vega-Lite is rapidly becoming a popular tool for creating charts as it has been incorporated into Jupyter Notebooks [9] and Python more generally through Altair [1]. Thus, developers who are familiar with Vega-Lite can form search queries without having to learn a new syntax and for those unfamiliar with Vega-Lite, our interface can help them learn a useful representation.

Note however, that unlike Vega-Lite which requires users to provide a complete specification to produce a chart, our query syntax allows partial specification. Queries may include only one or more aspects of the data, marks, encodings, axes, and non-data-encoding attributes of marks. Our query syntax also includes functional operators (e.g. count, gt, lt) and logical operators (e.g. and, or, not) on values and supports CompassQL's [47] syntax for replacing concrete values with wildcards.

Data: Queries about data can be based on the name of the data field or on data values. For example, users can search for visualizations the include a data field name "population" with the query

\{"data":\{"field": "population"\}\}

Although Vega-Lite does not include the "field" key as part of the "data" specification, it does include "field" as part of an 


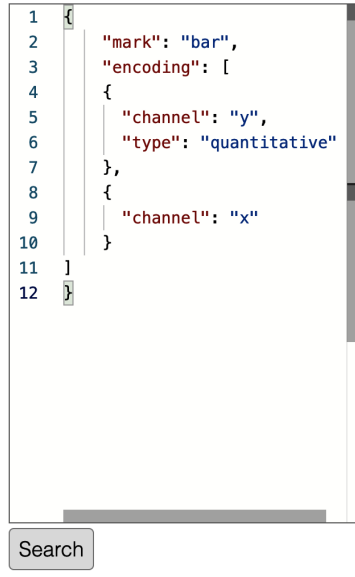

http://bl.ocks.org/lmatteis/raw/efd9be8f472e673eef6ce9d1951256a9/

\begin{tabular}{|l|l|l|l|}
\hline key & value & _deriv_width_6 & -deri \\
\hline female & 140600 & 0 & 1 \\
\hline male & 247000 & 1 & 0 \\
\hline & & & \\
\hline
\end{tabular}
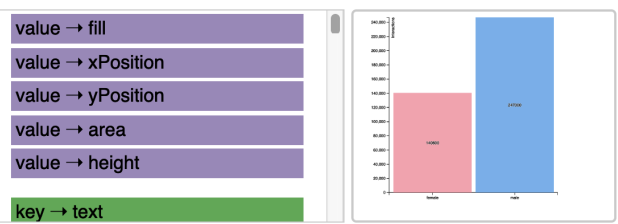

https://www.theatlas.com/charts/ByTqixXL

\begin{tabular}{|c|c|c|c|}
\hline colorIndex & entry & value & prev \\
\hline 0 & 883612800000 & 129.505 & 0 \\
\hline 0 & 915148800000 & 123.061 & 0 \\
\hline 0 & 946684800000 & 125.734 & 0 \\
\hline 0 & 978307200000 & 149.338 & 0 \\
\hline
\end{tabular}
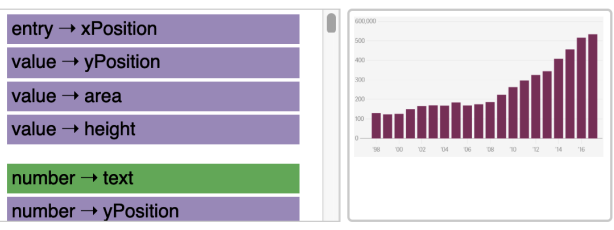

Fig. 4. Query for charts containing one quantitative data field using the y-position and another data field of either quantitative or nominal data type using the x-position of the bars. The search results are presented in list view showing the data table and encodings between data fields and mark attributes. The URL for the webpage containing the chart is given above the data table and a thumbnail shows the visual form of the chart and this query returns a set of vertical bar charts. As in Harper and Agrawala 2627 each encoding is represented as dfield $\rightarrow$ mattr and the background color indicates the data type: green for nominal data and purple for quantitative data. Users can click on 'Show more' button to expand the view so that all the data tables and encodings are visible.

"encoding" specification (Figure 36). We allow "field" as part of a "data" specification to enable queries based on field names that may be present in the dataset but not encoded in the corresponding visualization. Also note that our syntax allows any concrete string to be specified as a regular expression. For instance, we could replace "population" with ".*population.*” to find all visualizations that include a data field with the word "population" in the field name.

Our query syntax also allows users to apply functional operators on data values including ' "count', which computes the number of data values, statistical calculations on quantitative values ' 'min', ' 'max', ' 'sum' ' and ' 'average' ' and comparisons (' 'gt',',

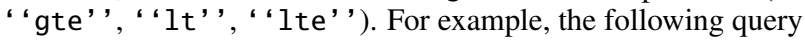

\{"data": \{"values": \{"count": \{"gte": 1000$\}\}\}$

returns visualizations containing at least 1000 data points. Finally, our syntax allows users to apply logical operators on an array of values. For example, the query



finds visualizations that have a data field named "country" with values including both "India" and "China".

Marks: Queries about marks can be based on the mark type. For example, the query $\{$ "mark": "circle" $\}$ would find all visualizations that include marks of type circle. Note that while D3 does not explicitly include the mark type bar, Vega-Lite does include it to enable concise specification of bar charts. Like Vega-Lite, we also consider bar as a mark type. Specifically after deconstruction, we convert marks of type rect that encode quantitative data using length and maintain a fixed width, to marks of type bar.

Encodings: Queries about encodings can be based on the dataencoding mark attribute (called ' 'channel' ' in CompassQL [47]), the data type or the data field name. Queries specifying a “ 'channel', can search for encodings using any data-encoding mark attribute value including color (i.e. fill-color), opacity, shape, text, angle, size (i.e. area), $x$-position or $y$-position. With this syntax, the query

\{"mark": "bar",

"encoding": [

\{"channel": "y", "type": "quantitative", "field": "*"\},

\{"channel": "x","type": "*", "field": "*"\} ]\}

finds all charts that encode a quantitative data field using the y-position of bar marks and encode another data field using the x-position of the bars. As in CompassQL, the “ " * ' ' symbol is a wildcard indicating that the data field encoded using y-position can have any data field name.
Similarly, the data field encoded using x-position can have any data field name and be of any data type. Note that to reduce the verbosity of our syntax, if the user does not include a complete Vega-Lite specification we treat the unspecified part of the query as a wildcard by default. Thus we could equivalently write this query as



As shown in Figure 4 this query retrieves vertical bar charts.

In addition to wildcards, our query syntax supports pattern variables. These variables start with the symbol $\$$ and can be used in multiple places to indicate that the search engine should bind the same value in each place it appears. For example, the query

\{"encoding": [

\{"channel": "x", "field": "\$t", "type": "quantitative"\}, \{"channel": "color", "field": "\$t", "type":"*"\}]\}

finds visualizations in which the same data field is redundantly encoded using the x-position and fill color of a mark. If we replace the pattern variable ' " $\$ \mathrm{t}$ ' ' with a wildcard “ "*, ' the query would return visualizations in which either the same or different data fields are encoded using x-position and color respectively.

Axes: In Vega-Lite an encoding may also be used to create and specify properties of axes. For example the syntax

\{"encoding": [\{"channel": "x", "axis": true\}]\}

can be used in our search engine to find visualizations that contain an x-axis. Users can similarly add axis properties to the query. For instance, adding "orient": "bottom" to the axis encoding would find charts containing an $\mathrm{x}$-axis near the bottom of the visualization.

Non-data-encoding attributes: To search for charts containing specific non-data-encoding attributes users can provide queries like \{"mark": \{"type": "text", "typeface": "Verdana"\}\} In this case, the query finds visualizations that contain at least one mark of type text that uses the " 'typeface' 'Verdana.

Metadata: In addition to the properties of visualization users can search the metadata of the webpages that contain the visualizations. Our query syntax supports keyword search on the surrounding webpage text (using "keyword',), webpage '“title', ' 'url',, “'domain', name, and the " 'timestamp', of the webpage if it contains one in its header. For example, the query

\{"domain": ". *nytimes.*"\}\}

finds all visualizations, where domain contains "nytimes". 

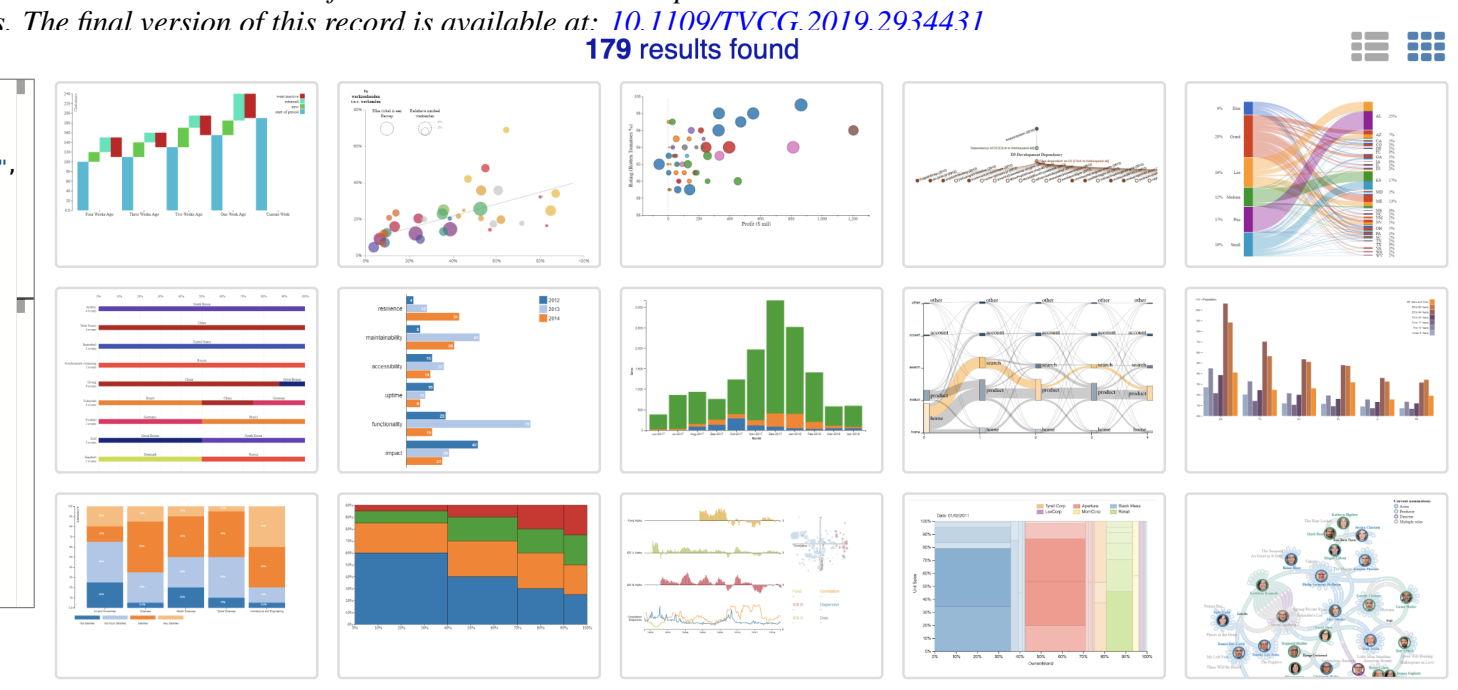

Fig. 5. Query for charts containing at least one encoding of nominal data fields and at least two encodings of quantitative data fields. Our search engine returns stacked and grouped bar charts, bubble charts and other variations with the desired encodings.

\subsubsection{Search Engine Interface}

The interface to our search engine includes a query textbox and either a grid of thumbnails view (Figure 1) or list view (Figure 4) of results. The interface initially shows the query in Figure 4 as an example of our query syntax that users can modify to formulate their own query. This design helps users who are learning the Vega-Lite syntax by supporting recognition of the syntax rather than having to recall it. To further help users quickly formulate queries our query textbox provides automatic code-formatting, auto-completion and validation using Visual Studio Code [18], an open source code editor library we access via a JavaScript wrapper. Visual Studio Code allows us to associate a schema describing the allowable values for each key in our query syntax (e.g. "channel", "field", "type"). Our interface then triggers auto-complete suggestions as the user is typing based on this query syntax scheme. It also validates the query by checking the structure and values in the query with respect to the schema and then highlights potential errors.

Our search interface can display the search results using two different ordering strategies. The ranking strategy counts the number of encodings in the search results that match the encodings explicitly specified in the query. We consider a pair of encodings to match if they both map data of the same data type to the same visual attribute of the same mark type. We rank the search results based on the number of matched encodings. If there is a tie in the number of matched encodings we consider how many encodings were unmatched, where more unmatched encodings indicates less relevance. Since search results generally match all of the explicit encodings in the query, this approach primarily relies on the tie-breaking approach to produce the final ordering. The randomized strategy randomly orders the search results and is designed to show users a variety of different designs that match the query. We use the randomized strategy by default.

\section{Applications}

Our search engine currently supports two main classes of applications: (1) design space exploration, and (2) information seeking.

\subsection{Design space exploration}

Often there are many ways to visualize the same dataset. In order to find a representation that is effective and expressive, visualization developers typically explore a variety of design choices. Our search engine supports developers in exploring the design space by enabling a range of queries based on visual style and structure of D3 charts.

Data, marks, and encodings: Given a dataset with a particular combination of data types, visualization developers often need to consider the possible alternatives for depicting the data. For example, suppose a dataset contains one nominal and two quantitative data fields. A developer might issue the query shown in Figure 5 to find charts that encode at least one nominal and two quantitative data fields. In this query the count and gte operators are only necessary when querying for more than one encoding of the same type. A query for "encoding": [ "type": "nominal" $\}]$ would return charts that encode at least one nominal data field.

Users can search for different basic chart types (e.g., bar charts, scatterplots) by querying for combinations of encodings. For example, to retrieve bar charts, the query in Figure 4 looks for charts that encode quantitative data using the y-position attribute of bars and contain both $\mathrm{x}$ - and $\mathrm{y}$-axes. Similarly, the query in Figure 1 looks for scatterplots that encode an additional data fields using the color of circle marks.

Users can also query based on the values of the data fields. Consider a visualization developer who has a quantitative data field with both positive and negative values and wants to create a bar chart with a baseline at zero, such that the positive bars extend upwards (or to the right for a horizontal bar chart) and negative bars extend downwards (or to the left). A novice developer may find it tricky to implement such a bar chart because rectangles in D3 are positioned using their top-left corner and cannot have negative heights or widths. Using our search engine the developer can query to find a variety of bar charts where both positive and negative values are encoded as in Figure 6 The query looks for charts that map a quantitative data field to either the $\mathrm{x}$ - or y-position of bars. The min and max conditions indicate that the data field depicted in the chart should have both negative and positive values. The pattern variable ' ' $\$ t$ ', is used to ensure that the same data field containing negative and positive values is encoded using the $\mathrm{x}$ - or y-position attributes. The resulting bar charts include a number of stylistic variations of bar charts with negative and positive values.

Developers sometimes face the problem of visualizing large datasets containing thousands of data points in limited screen space. In such cases, It can be useful to see a gallery of example visualizations to see how other visualization developers dealt with similarly large datasets. With the query shown in Figure 7 our search engine returns charts in which at least one quantitative data field contains at least 1500 data points encoded using circle marks. Since the "channel" is not specified in the query, any possible visual attribute (e.g. positions, area, and color) may be used to encode the data.

Query by example: Developers sometimes seek visualizations that are similar to an example visualization. For example, suppose a visualization developer is interested in seeing the stylistic variations amongst bar charts that use the fill-color of the bars to depict an additional nominal data field. Alternatively, the developer may wish to examine different ways to implement this type of bar chart. Our search engine lets users select an example visualization - e.g. a bar chart with an additional fill-color encoding - and returns similar visualizations based on the similarity of their marks and encodings as in Figure 8

To implement this functionality, when users provide an example chart as the query, our system deconstructs it (Section 3.2) and then forms a query consisting of the mark and encoding specifications found 

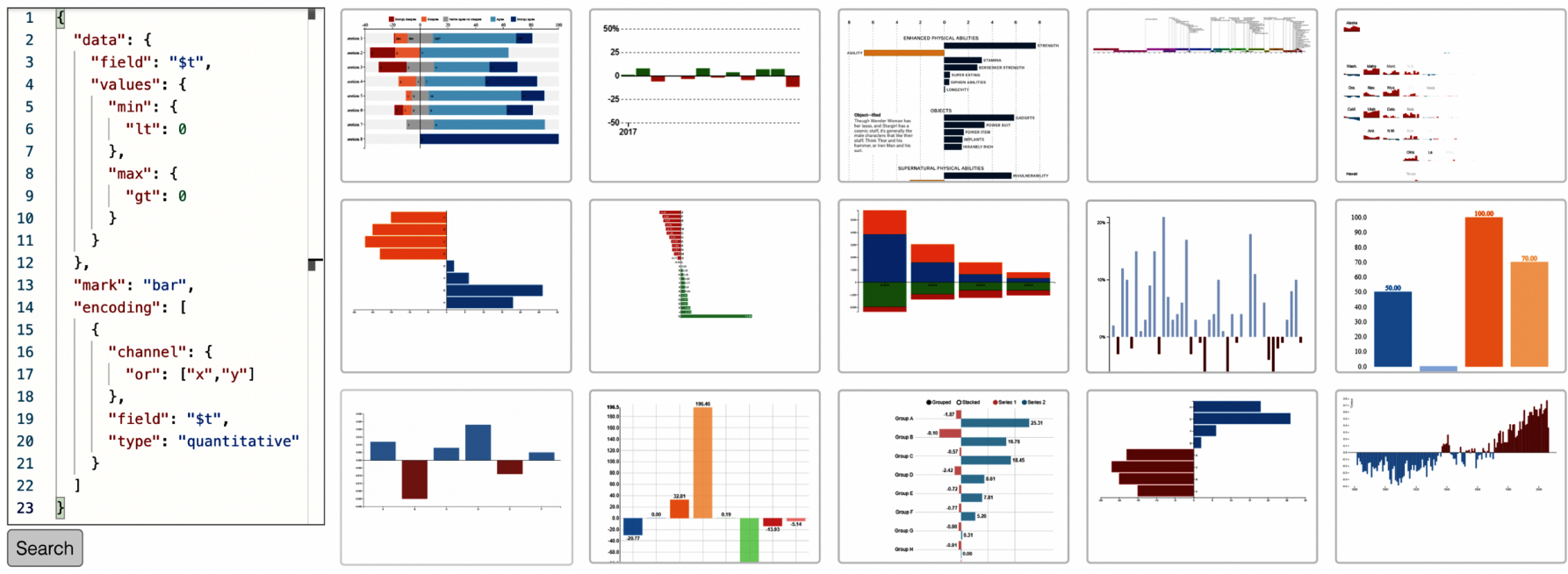

Fig. 6. Query for charts containing a quantitative data field with both negative and positive data values mapped to the $x$ - or $y$-position attribute of bar marks. Our search engine mostly returns bar charts with bars extending in both directions from a baseline at zero. The user could filter out this result by for example including another condition in the query checking for axes.

Query





42 results found

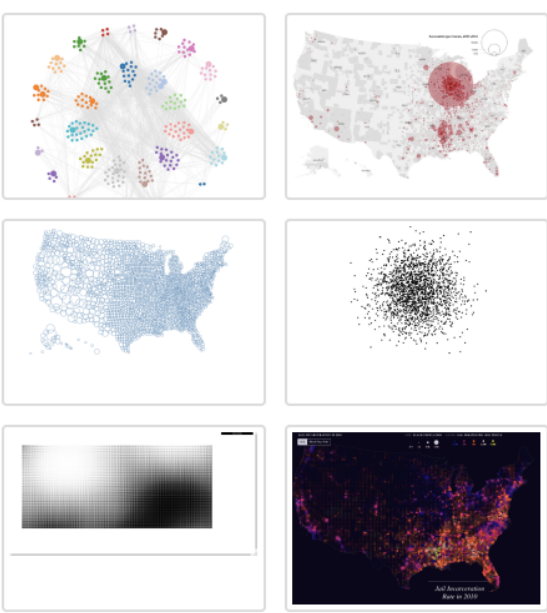

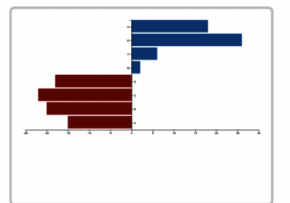

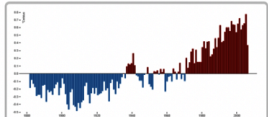

Fig. 7. Query for visualizations that contain at least 1500 data points of a quantitative data field encoded using some visual attribute of circle marks. The results include scatterplots, bubble charts and other more exotic chart types.

in the example. However, the query omits data field names, axis properties and non-data encoding mark properties to allow variations in the results set. Thus, users can see stylistic variations (e.g., different background colors, typefaces or usage of grid-lines) in the thumbnail view and go to the source webpage to examine the underlying code.

Query by non-data-encoding attributes: Charts often include visual attributes that do not encode data but are used for styling such as the background color, typefaces, stroke-widths, etc. Consider for example, a developer who wants to create a chart with dark background and needs to find suitable colors for filling the foreground data-encoding marks. The query in Figure 9 finds charts where the background color is similar to the named color "slategray" and that contain foreground data-encoding marks that encode data using fill-color. We use the functional operator "colorSim" to compute the color similarity as the distance between the background color of each chart in our database and "slategray" in LAB color space. When using this operator our search engine rank orders the resulting charts based on this distance and the developer can see how the color choices affect the visual design.

\subsection{Information seeking}

Sometimes users wish to find visualizations that are related to a specific topic. For example, a data journalist may seek charts about the latest US election. While keyword-based search engines like Google or Sightline [42] can return some visualizations on such topics, our search engine further enhances the information seeking process by complementing text-based search with retrieval based on mark and data attributes. For example, the query

\{"keyword": "US election", "encoding": [\{

"channel": "color", "type": "nominal",

"values": \{"and": ["red", "blue"]\} \}]\}

finds charts that appear on webpages containing the word "US election" and that use blue and red fill-colors to encode nominal data (Supplemental Materials: Figure 2).

Our search engine also lets users find visualizations based on data field names and titles. However, sometimes a data field may not have the exact same name that the user specified and instead use a semantically similar synonym. For such cases we provide the "wordSim" operator which computes semantic similarity to any given word. Specifically, we use a word2vec vector model [33] pre-trained on parts of the Google News dataset [20] and calculate the cosine distance between the word2vec representation of the given word and other words in our chart database. For example,

\{"data":\{"field": \{"wordSim": "population"\}\}\}

computes the distance between the word2vec representation of data field name "population" and each of the data field names in our corpus 

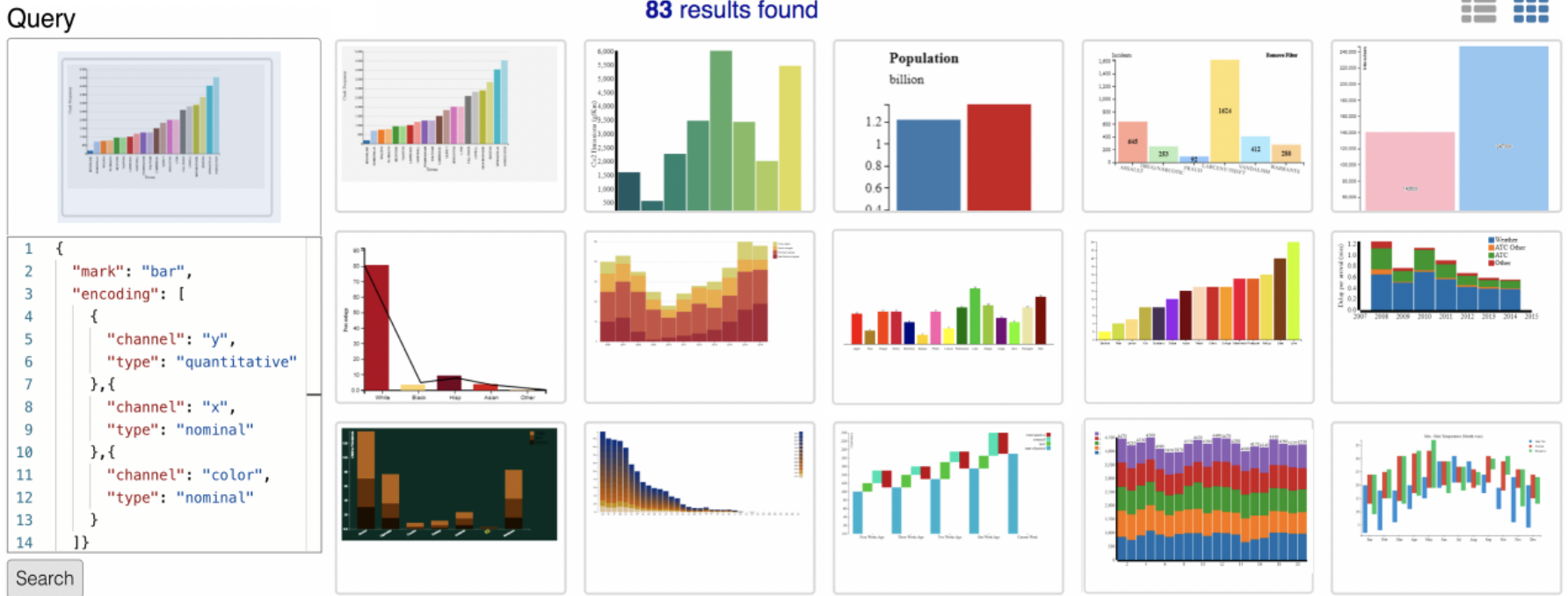

Fig. 8. In query by example users select a chart returned by an earlier query (shown at top of query textbox) and our search engine finds charts with similar encodings. Here, the example query is a vertical bar chart that contains an additional encoding that maps a nominal data field to the fill-color of the bars. Our search engine automatically converts the example into our query syntax keeping only the encodings (shown at bottom of query textbox). The query returns a variety of vertical bar charts that match as many of the encodings as possible.

Query

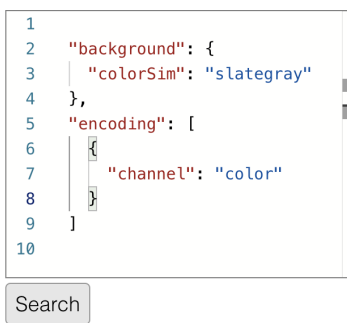



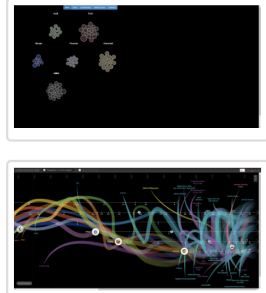

35 results found



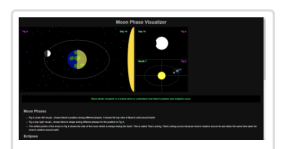

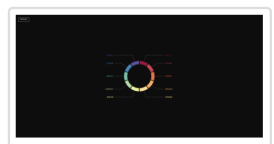



Fig. 9. Query for charts containing dark backgrounds similar in color to "slategray" and that encode data using the fill-color of foreground marks. The results include a variety of color palettes for the foreground marks.

of visualizations. We consider the data field to be relevant whenever the cosine similarity between them is greater than a threshold $\tau$, which we empirically set to 0.75. As shown in Supplemental Materials: Figure 3 , the charts resulting from this query are all related to population but contain data fields named "population", "people", and "human".

\section{Design Demographics analysis}

We have used our search engine to perform a large scale demographic analysis across our collection of 7860 D3 visualizations. For comparison, we independently analyze the subcollection of 457 D3 charts we crawled from the New York Times. Our goals in conducting these analyses is to identify patterns of visual design that are commonly used by D3 chart developers and to show how other researchers might use our search engine to examine visualization collections at scale. Our analysis suggests that visualization developers often adhere to best design practices as they create D3 visualizations, even if they only implicitly know these practices.

Use of marks: We analyze the number of charts using each mark types to encode data (Figure 10a). Across our whole collection of D3 charts we find that path marks are most frequent (25.0\%), as they are commonly used to generate line charts and the regional boundary shapes (e.g. state boundaries) used in choropleth maps. Text marks $(21.2 \%)$ are used to show the data in text form. Circle marks (18.2\%) are commonly used in scatterplots. Rect marks (14.6\%) are used for heatmaps, treemaps, and legends, while bar marks (13.7\%) appear exclusively in bar charts. Line marks (10.4\%), arc marks $(2.3 \%)$ as used in donut and pie charts, polygon marks (0.6\%) and ellipse marks $(0.2 \%)$ are used to encode data far less frequently. Our mark type frequencies are consistent with Battle et al.'s [21] chart type analysis of 1247 D3 charts, who find that map charts (30.4\%), line charts (12.6\%), bar charts $(12.3 \%)$ and scatterplots $(9.46 \%)$ are the four most common types of D3 charts while pie charts, donut charts and radial charts appear infrequently (together $<0.05 \%$ ).

In our New York Times subcollection (Figure 10 a right) we find a different pattern of mark usage; they use paths (34.1\%) far more often than the other marks and use far fewer circles $(5.9 \%)$, rects $(5.0 \%)$ and bars $(4.8 \%)$. Manually inspecting the New York Times subcollection, we find that it contains fewer scatterplots, heatmaps, treemaps, and bar charts than the collection as a whole, but often includes more elaborate, and unconventional charts such as maps, networks and trees which typically involve lots of paths.

Use of mark attributes: We also analyze the frequency with which different mark attributes are used to encode quantitative and nominal data (Figure 10p). Across both collections (Whole and New York Times) we find that the pattern of attribute use to encode quantitative data is similar and similar to the perceptual effectiveness rankings of visual attributes proposed by Mackinlay [32]. He suggests that position is the most effective encoding for quantitative data, followed by width and height, and then angle, area, fill-color, and opacity. Although we find more area encodings in our collections than either height or width we note that bar charts redundantly encode data using either height (or width) and area - because the non-data-encoding size attribute of the bars (either width or height) is fixed making area proportional to the data-encoding attribute. If we combine the usage of height and width encodings we find they are used more often than area encodings and in-line with Mackinlay's rankings. These findings suggest that D3 developers may be aware (perhaps implicitly) of the perceptual effectiveness rankings when they are designing charts.

Use of multiple encodings: Visualization developers sometimes choose to encode multiple data fields using different attributes of the same mark (Supplemental Materials: Figure 4a). Across our whole collection, we find that while some data-encoding marks (27.8\%) are used to encode a single data field the majority of such marks (72.2\%) encode multiple data fields. Using two or more mark attributes to encode data 


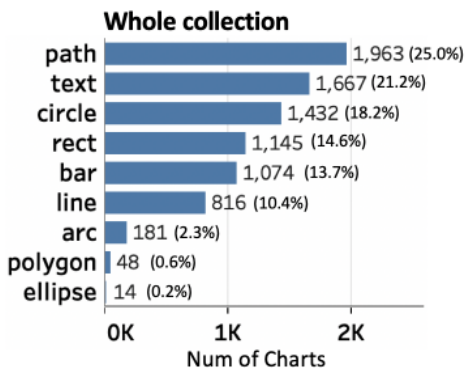

(a) Use of each mark type to encode data

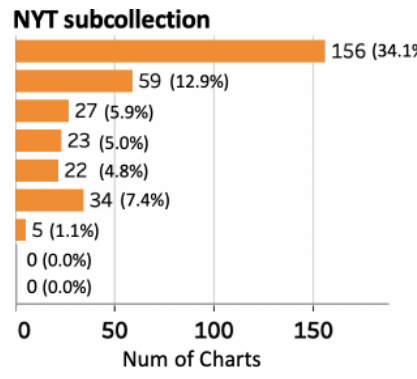

mark type encode data

Fig. 10. Number of charts using each mark type for encoding data (a). The percentages are with respect to the total number of charts in each collection (7860 whole collection, $457 \mathrm{New}$ York Times subcollection). Note that some charts contain multiple data encoding marks while others do not contain any encodings. Number of times each mark attribute is used to encode quantitative or nominal data (b).

is far more frequent for circles than for any other mark type, as developers often use fill-color and area in addition to position to encode data in scatterplots and bubble charts. While two or three encodings per mark is quite prevalent, occurrences of five or more encodings per mark is rare (less than $1 \%$ of marks in our whole collection), as such designs can make it difficult to interpret the chart. The pattern of using multiple encodings is similar in our New York Times subcollection (Supplemental Materials: Figure 4a right).

We also analyze which pairs of visual attribute developers commonly use when encoding multiple quantitative data fields using the same mark (Supplemental Materials: Figure 4b). We find that they primarily follow the approach of using the most perceptually effective visual attributes [32]34] for encoding quantitative data - i.e. combinations of $x$-, y-position, area, width and height are most prevalent. Again, the pattern is similar for the New York Times subcollection (Supplemental Materials: Figure $4 b$ right).

Use of integral/separable attribute pairs: One challenge with multiple encodings is that one encoding may interfere with the perceptual effectiveness of another encoding. Graphical perception researchers call pairs of attributes integral if viewers have difficulty reading one without interference from the other and call them separable otherwise [35 45]. To avoid interference attributes that are integral should ideally encode data that is highly correlated while attributes that are separable can encode correlated or uncorrelated data. Supplemental Materials: Figure $4 \mathrm{c}$ shows for each pair of data-encoding attributes, how well the corresponding data fields are correlated. We find that the Pearson correlation coefficients cover the whole range of possible values for the first three pairs of attributes. For many of the pairs lower in the list, the encoded data fields tend to either be uncorrelated (near 0 correlation) or highly correlated (near 1 or sometimes -1 ) with a few scattered examples in between. Pairs of attributes that are known to be highly integral such as width and height are primarily used to encode highly correlated data fields. The pattern is even stronger for our New York Times subcollection (Supplemental Materials: Figure 4c right) These results suggest that visualization developers, especially at the New York Times, typically follow good practices of using integral pairs of encoding attributes when the data fields are correlated.

Use of fill-color encodings: Visualization developers and researchers have established best practices for using fill-color to encode data. For example, a rule of thumb is to limit the number of colors used to encode nominal data to between six and twelve, so that viewers can perceptually discriminate between them [34 45]. Using our search engine we find that among the visualizations that use fill-color to encode nominal data, $58.0 \%$ use six or more different colors while $13.6 \%$ use twelve or more different colors (Supplemental Materials: Figure 5). However, in the New York Times subcollection, we find that there was not a single chart using eight or more different colors to encode nominal data but that $47.9 \%$ use six or more different colors. These results suggest that in practice developers commonly use 6 or more colors for nominal encodings, but avoid using 12 or more colors.

\section{USER Study}

To better understand the effectiveness of our search engine at helping developers explore the style and structure of visualizations, we conducted a user study to compare it with a baseline interface (Sightline [42]) that allows keyword search over webpages containing D3 visualizations, but not over the data, marks, encodings and style attributes.

\subsection{Study Design}

We designed a comparative, within-subjects study with twelve participants (ages 18 to 44, four female). They were data analysts, professional developers, and students who develop data visualizations frequently as a part of their work to explore data and present their findings. All participants were experienced with visualization development using programmatic tools like D3 [6], Vega-Lite [40], R/ggplot2 [7], and Matplotlib [10]. They also reported that they frequently use chart construction software like Microsoft Excel [11] and Tableau [16].

Each participant performed two exploratory search tasks, one with our search engine interface and one with the baseline interface (Sightline [42]), in counterbalanced order across participants. Each task consisted of a scenario in which the participant would work as a visualization developer and needed to find five visualizations that matched some design criteria. For instance, in one task, participants were given a dataset with one quantitative and two nominal data fields and they were asked to look for five different charts that might be suitable for showing that dataset. They were told that the five results should serve as design inspirations and that they should look through the corresponding code to consider how they might reuse or adapt it to their own design.

Each participant completed a pre-study background questionnaire. We then gave a brief introduction explaining the capabilities of two search interfaces. For our search interface, we introduced the query syntax and explained how to specify search over data, marks, encodings, axes and non-data-encoding attributes as described in Section 3.3 Participants could also access a tutorial page with a written description of the query syntax at any time. After completing each task, we asked participants to complete a post-task questionnaire. The study lasted approximately 45 minutes and each participant was paid $\$ 15$.

\subsection{Results}

In the post-task questionnaires, participants rated each interface (baseline and ours) with respect to five measures (Usefulness, Ease of use, Satisfaction, Relevance, Specificity) on 5-point Likert as shown in Figure 11. Our interface received significantly higher ratings on three of the five measures: Usefulness (Mann-Whitney $U=37 ; p=0.035$ ), Satisfaction $(U=37 ; p=0.027)$ and Specificity $(U=38 ; p=0.031)$. For the Relevance measure, our interface received a higher rating overall, but the difference was not significant $(U=52 ; p=0.223)$. There was no significant difference between the rating for ease of use $(U=57 ; p=0.342)$. We also found no significant difference in the task completion time for our interface $(M=324 \mathrm{sec}$., $\mathrm{SD}=48)$ and the baseline $(\mathrm{M}=311 \mathrm{sec} ., \mathrm{SD}=41)$ conditions according to the t-test; $\mathrm{t}(22)=0.68, \mathrm{p}=0.501$. Overall in comparison to the baseline, these results suggest that our participants found our search interface to be more useful, they were more satisfied with our search results and that it enabled them to find examples that match specific chart criteria.

Most participants started with simple queries based on mark type and one or two encoding criteria. They primarily used the thumbnail view of the interface for browsing the results and occasionally clicked on the result items for further examination and to visit the webpage 


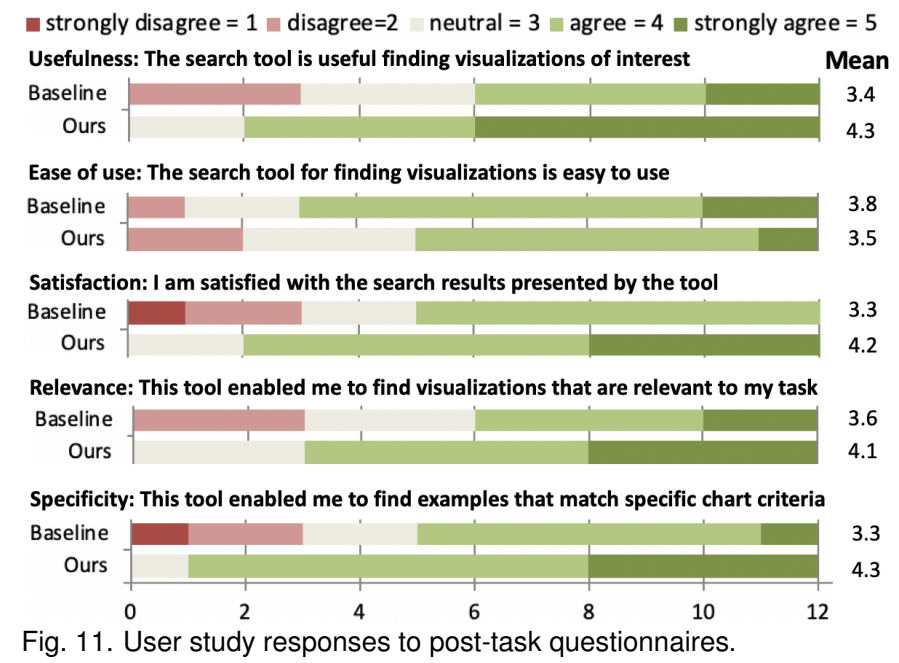

from where the visualization was crawled. Once they became more familiar with the query syntax, they sometimes added more criteria (e.g. non-data encoding attribute such as background) to fulfill their specific search needs. While some participants had difficulty in formulating the query (especially at the beginning), gradually they became comfortable with the help of the auto-complete suggestions triggered by our search interface as well as by accessing the tutorial. Four participants used the query-by-example feature; they initially gave a text query but then selected a result chart that looked relevant and submitted it as a query to find other similar charts. For the baseline, participants usually started with basic keywords focused on chart types they thought might be useful for the task (e.g. 'bar', 'stacked bar', 'scatter plot'), and then scanned through the paginated list of results. But because chart type does not always appear in the surrounding webpage text or its metadata, the baseline interface only returned a subset of charts of the given type.

In an open-ended feedback session, participants told us that using our tool they were able to find a variety of charts that were relevant to the given task. Overall, they felt that our search engine enabled them to find charts based on design criteria and this would save them hours of time in chart design and implementation. One participant mentioned "I sometimes spend nearly a whole day to find ideal example visualizations to use their code for my visualization development task, but this tool can exactly solve that problem within minutes." Another participant said, "I am satisfied in finding so many cool visualizations on the Web and now I can really use them in my day to day work."

The participants also identified a couple of areas of improvement for our interface. A few participants felt that they needed time to get familiar with the query syntax and that coming up with queries that represented their complex design goals was sometimes challenging. One participant suggested providing a more diverse gallery of query examples in the tutorial page to provide further help in formulating the query and another participant suggested to introduce faceted search techniques for filtering search results more easily.

\section{LIMITATIONS}

While our search engine for D3 visualizations demonstrates a broad range of applications and enables large-scale demographic analysis, it does have some limitations.

Limitations of D3 deconstructor. The deconstruction tool we have extended from Harper and Agrawala [26 27] can accurately extract the data, marks and encodings from a variety of basic D3 charts including variants of bar charts, scatterplots, line charts, etc. However, it cannot fully recover certain types of complex encodings such as non-linear function mappings (e.g., logarithmic scale) between data and mark attributes. In other cases, charts may use complex algorithmic techniques for encoding the data (e.g., force-directed layout algorithm) and their deconstructor cannot recover such mappings. Our deconstructor inherits these limitations. But despite these issues, our search engine does enable exploration over a broad range of the visualization design space for the most commonly used charts and graphs.
Limitations of indexing and retrieval. Our system indexes D3 visualizations based on their data, marks, and encodings, axes, non-dataencoding attributes as well as the page text surrounding the visualizations. However, we do not currently index the JavaScript code that constructs the visualization. Automatic analysis of D3 code could provide additional ways of indexing and querying the visualizations. For example, developers might benefit from finding pieces of code where a particular D3 function appears in order to learn how to use it in different contexts. This approach may also allow our search engine to index and retrieve visualizations that exhibit a particular interactive technique and/or animation. Recent work on finding API usage in programming code [25] could provide a starting point along this direction.

Limitations of query syntax. While our query syntax is designed for visualization developers who are familiar with mapping-based specifications (e.g. Vega-Lite), novice users may have difficulties expressing their design needs using our current query syntax. Introducing natural language queries could make it easier for such users to find visualizations. For example, given a query (e.g. "vertical bar charts") the system might automatically generate the corresponding Vega-Lite specification for it and then apply the query to retrieve the corresponding visualizations. Seeing the query in the Vega-Lite syntax could help the user understand the conversion and allow for further refinement of the query as necessary. However, converting the natural language query into our query syntax could also introduce new challenges (e.g. ambiguity in natural language could open up multiple possible interpretations). Another direction for supporting novice users is to enhance our queryby-example feature, where the interface might allow users to select a template visualization and then modify the template by changing styles and visual encodings, as demonstrated by Saket et al. [39], to find visualizations that match their desired template.

Limitations of user study design. Due to the exploratory nature of search tasks, we focused our user study on collecting and analyzing subjective data. While such subjective data does provides information about perceived usefulness, a set of objective metrics such as time to construct the query, time to complete the search, the variety of charts found and accuracy (e.g. precision) could complement the results and is an important direction for future work. Longitudinal studies over an extended period of time would also help us further understand the usefulness and adoption rate among real users in the future.

\section{Conclusions ANd Future wORK}

We have presented a search engine that indexes and retrieves D3 visualizations based on the data, marks, encodings, axes, non-data-encoding attributes and webpage metadata. We demonstrate that the resulting search engine enables visualization developers to explore the visualization design space and find visualizations that match certain data and design characteristics. Users can query our collection of D3 visualizations in a variety of new ways, encoding based search, to query by styles to query by example. We believe that this work is an initial step towards searching and analyzing visualizations at-scale based on their data and their visual encodings. Our search engine system is available at https://www.yorku.ca/enamulh/vis-search.

There are several avenues for future work. Extending the deconstructor to handle other SVG-based visualizations such as those generated by Plotly [15], Chartblocks [5] and Graphiq [8], or indexing the collection of charts used in Beagle [21] would further enrich our collection and enhance the generalizability of our search engine. The number of Vega-Lite [40] charts is growing rapidly and since our query syntax is based on this language already we plan to index these charts in the immediate future. There are also several collections of imagebased charts available in the research community such as MassVis [22], VizioMetrics [31] and FigureSeer [43]. Applying recent methods for deconstructing image-based charts would allow us to index and search through such large collection of visualizations [29 36 37]. Moreover, adding different diverse collections could help us to discover differences in design demographics patterns across a wider variety of sources.

\section{ACKNOWLEDGMENTS}

The authors thank Dae Hyun Kim for feedback over the course of this work. This work was supported by NSF award III-1714647. 


\section{RefERENCES}

[1] Altair, 2019. Retrieved March 20, 2019 from https://altair-viz github.io/

[2] Big list of D3 examples, 2019. Retrieved March 20, 2019 from http: //christopheviau.com/d3list/

[3] Blocks, 2019. Retrieved March 20, 2019 from https://bl.ocks .org

[4] Bloomberg, 2019. Retrieved March 20, 2019 from https://www bloomberg.com/graphics

[5] Chartblocks, 2019. Retrieved March 20, 2019 from https://www chartblocks.com/

[6] D3.js, 2019. Retrieved March 20, 2019 from https://d3js.org

[7] ggvis, 2019. Retrieved March 20, 2019 from https://ggplot2 tidyverse.org/

[8] Graphiq, 2019. Retrieved March 20, 2019 from https://www . graphiq com/

[9] Jypyter Notebook, 2019. Retrieved March 20, 2019 from https:// jupyter.org/

[10] Matplotlib, 2019. Retrieved March 20, 2019 from https://matplotlib org/

[11] Microsoft Excel, 2019. Retrieved March 20, 2019 from https:// products.office.com/en-ca/excel

[12] MongoDB, 2019. Retrieved March 17, 2019 from https://www mongodb.com/

[13] New York Times, 2019. Retrieved March 20, 2019 from nytimes.com/ interactive/

[14] New York Times Interactive, 2019. Retrieved March 20, 2019 from http://www.nytimes.com/newsgraphics/2013/12/30/ year-in-interactive-storytelling/index.html

[15] Plotly, 2019. Retrieved March 20, 2019 from https://plot.ly/

[16] Tableau Software, 2019. Retrieved March 20, 2019 from https://www tableau.com/

[17] The Pudding, 2019. Retrieved March 20, 2019 from https://pudding $\mathrm{cool} /$

[18] Visual Studio Code, 2019. Retrieved March 17, 2019 from https:// code.visualstudio.com/

[19] Wall Street Journal, 2019. Retrieved March 20, 2019 from http:// graphics.wsj.com

[20] Word embedding trained on Googole News, 2019. Retrieved March 17, 2019 from/https://code.google.com/archive/p/word2vec/

[21] L. Battle, P. Duan, Z. Miranda, D. Mukusheva, R. Chang, and M. Stonebraker. Beagle: Automated extraction and interpretation of visualizations from the Web. In Proc. of SIGCHI, pp. 594:1-594:8, 2018

[22] M. A. Borkin, A. A. Vo, Z. Bylinskii, P. Isola, S. Sunkavalli, A. Oliva, and H. Pfister. What makes a visualization memorable? IEEE Transactions on Visualization and Computer Graphics, 19(12):2306-2315, 2013.

[23] Z. Chen, M. Cafarella, and E. Adar. DiagramFlyer: A search engine for data-driven diagrams. In Proc. of WWW, pp. 183-186, 2015.

[24] M. Cliche, D. Rosenberg, D. Madeka, and C. Yee. Scatteract: Automated extraction of data from scatter plots. In Conference on Machine Learning and Knowledge Discovery in Databases, pp. 135-150, 2017.

[25] E. Glassman, T. Zhang, B. Hartmann, and M. Kim. Visualizing API usage examples at scale. In Proc. of SIGCHI, pp. 580:1-580:12, 2018.

[26] J. Harper and M. Agrawala. Deconstructing and restyling D3 visualizations. In Proc. of UIST, pp. 253-262. ACM, 2014.

[27] J. Harper and M. Agrawala. Converting basic D3 charts into reusable style templates. IEEE TVCG, pp. 1274-1286, 2017.

[28] J. Heer, J. Mackinlay, C. Stolte, and M. Agrawala. Graphical histories for visualization: Supporting analysis, communication, and evaluation. IEEE Transactions on Visualization and Computer Graphics, 14(6):1189-1196, Nov. 2008.

[29] D. Jung, W. Kim, H. Song, J.-i. Hwang, B. Lee, B. Kim, and J. Seo. ChartSense: Interactive data extraction from chart images. In Proc. of SIGCHI, pp. 6706-6717, 2017.

[30] R. Kumar, A. Satyanarayan, C. Torres, M. Lim, S. Ahmad, S. R. Klemmer, and J. O. Talton. Webzeitgeist: design mining the web. In Proc. of SIGCHI, pp. 3083-3092, 2013.

[31] P.-s. Lee, J. D. West, and B. Howe. Viziometrix: A platform for analyzing the visual information in big scholarly data. In Proc. of $W W W$, pp. 413418, 2016

[32] J. Mackinlay. Automating the design of graphical presentations of relational information. ACM Trans. on Grap. (TOG), 5(2):110-141, 1986.

[33] T. Mikolov, K. Chen, G. Corrado, and J. Dean. Efficient estimation of word representations in vector space. arXiv:1301.3781, 2013.

[34] T. Munzner. Visualization Analysis and Design. CRC Press, 2014.

[35] S. E. Palmer. Vision science: Photons to phenomenology. 1999.

[36] J. Poco and J. Heer. Reverse-engineering visualizations: Recovering visual encodings from chart images. In Computer Graphics Forum (Proc. EuroVis), vol. 36, pp. 353-363, 2017.

[37] J. Poco, A. Mayhua, and J. Heer. Extracting and retargeting color mappings from bitmap images of visualizations. IEEE Transactions on Visualization and Computer Graphics (Proc. InfoVis), 24(1):637-646, 2018.

[38] D. Ritchie, A. A. Kejriwal, and S. R. Klemmer. d. tour: Style-based exploration of design example galleries. In Proc. of UIST, pp. 165-174. ACM, 2011.

[39] B. Saket, H. Kim, E. T. Brown, and A. Endert. Visualization by demonstration: An interaction paradigm for visual data exploration. IEEE Transactions on Visualization and Computer Graphics, 23(1):331-340, Jan 2017.

[40] A. Satyanarayan, D. Moritz, K. Wongsuphasawat, and J. Heer. Vega-lite: A grammar of interactive graphics. IEEE Transactions on Visualization and Computer Graphics, 23(1):341-350, 2017.

[41] M. Savva, N. Kong, A. Chhajta, L. Fei-Fei, M. Agrawala, and J. Heer. Revision: Automated classification, analysis and redesign of chart images. In Proc. of UIST, pp. 393-402. ACM, 2011.

[42] J. Sechler, L. Harrison, and E. M. Peck. Sightline: Building on the web's visualization ecosystem. In Proc. of SIGCHI, pp. 2049-2055. ACM, 2017.

[43] N. Siegel, Z. Horvitz, R. Levin, S. Divvala, and A. Farhadi. FigureSeer: Parsing result-figures in research papers. In European Conference on Computer Vision, pp. 664-680. Springer, 2016.

[44] H. Stitz, S. Gratzl, H. Piringer, T. Zichner, and M. Streit. Knowledgepearls: Provenance-based visualization retrieval. IEEE TVCG, 2018

[45] C. Ware. Information visualization: perception for design. Elsevier, 2012.

[46] L. Wilkinson. The grammar of graphics. In Handbook of Computational Statistics, pp. 375-414. Springer, 2012.

[47] K. Wongsuphasawat, Z. Qu, D. Moritz, R. Chang, F. Ouk, A. Anand, J. Mackinlay, B. Howe, and J. Heer. Voyager 2: Augmenting visual analysis with partial view specifications. In Proc. of SIGCHI, pp. 26482659. ACM, 2017. 


\section{Supplemental Materials}

\section{Visualization Crawler}

Our visualization crawler extracts weblinks from a set of seed URLs, then downloads the corresponding webpage and finally renders the resulting page in the Chrome browser so that we can apply our visualization deconstructor to the page. Our crawler only follows one level of links, and we seed it with webpages containing lists of links to D3 visualizations. Members of the D3 developer community have compiled several such lists at sites like d3.js [3], bl.ocks.org [2] and the "Big List of D3 Examples" [1]. We supplement these community curated seed lists with additional lists of interactive visualizations that are available directly from news sites like the New York Times [5] and the Wall Street Journal [7].

In total we crawled 2623 webpages from 622 different domains, where each page contained at least one D3 visualization, and we deconstructed a total of 7860 D3 visualizations as described in Section 3. We also captured screenshots of the visualization as well as the HTML of the webpage it was embedded in. The resulting database takes 5.6 $\mathrm{GB}$ of disk storage. Figure 1 shows the top 20 domains in terms of the number of D3 visualizations we extracted from them.

\section{Applications: INFORMation SEEKING}

Sometimes users wish to find visualizations that are related to a specific topic. For example, a data journalist may seek charts about the latest US election. While keyword-based search engines like Google or Sightline [15] can return some visualizations on such topics, our search engine further enhance the information seeking process by complementing text-based search with retrieval based on mark and data attributes. For example, the query

\{"keyword": "US election", "encoding": [ [

"channel": "color", "type": "nominal",

"values": \{"and": ["red", "blue"]\} \}]\}

finds charts that appear on webpages containing the word "US election" and that use blue and red fill-colors to encode nominal data (Figure 2).

Our search engine also lets users find visualizations based on data field names and titles. However, sometimes a data field may not have the exact same name that the user specified and instead use a semantically similar synonym. For such cases we provide the "wordSim" operator which computes semantic similarity to any given word. Specifically, we use a word2vec vector model [12] pre-trained on parts of the Google News dataset [8] and calculate the cosine distance between the word2vec representation of the given word and other words in our chart database. For example,

\section{\{"data":\{"field": \{"wordSim": "population"\}\}\}}

computes the cosine distance between the word2vec representation of data field name "population" and each of the data field names in our corpus of visualizations. We consider the data field to be relevant whenever the cosine similarity between them is greater than a threshold

Manuscript received xx xxx. 201x; accepted xx xxx. 201x. Date of Publication $x x x x x$. 201x; date of current version xx xxx. 201x. For information on obtaining reprints of this article, please send e-mail to: reprints@ieee.org. Digital Object Identifier: $x x . x x x x / T V C G .201 x . x x x x x x x$

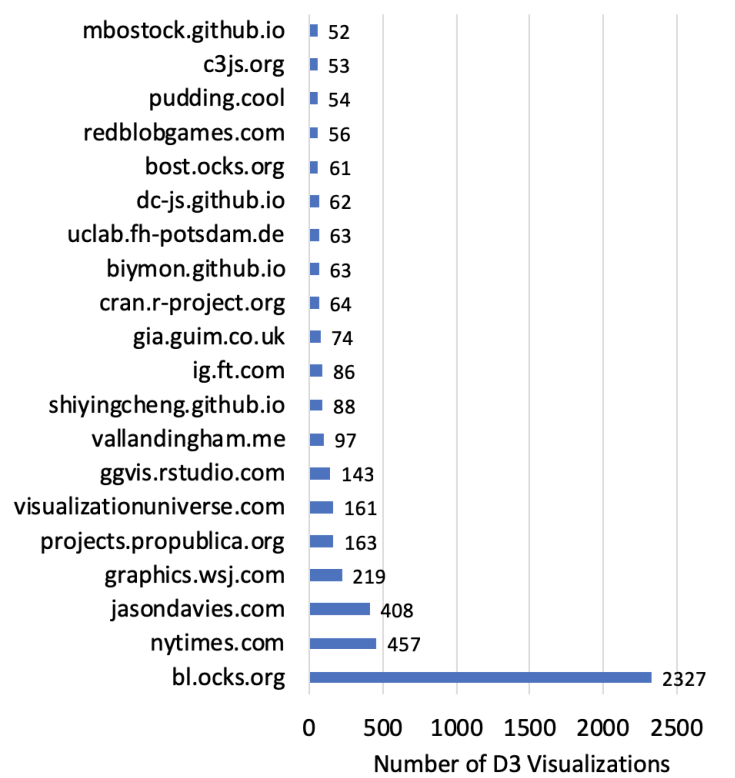

Fig. 1. Top 20 domains in terms of number of D3 visualizations we extracted from them.

$\tau$, which we empirically set to 0.75 . As shown in Figure 3 the charts resulting from this query are all related to population but contain data fields named "population", "people", and "human".

\section{Design Demographics Analysis}

Use of multiple encodings: Visualization developers sometimes choose to encode multiple data fields using different attributes of the same mark (Figure 4a). Across our whole collection, we find that while some data-encoding marks $(27.8 \%)$ are used to encode a single data field the majority of such marks $(72.2 \%)$ encode multiple data fields. Using two or more mark attributes to encode data is far more frequent for circles than for any other mark type, as developers often use fillcolor and area in addition to position to encode data in scatterplots and bubble charts. While two or three encodings per mark is quite prevalent, occurrences of five or more encodings per mark is rare (less than $1 \%$ of marks in our whole collection), as such designs can make it difficult to interpret the chart. The pattern of using multiple encodings is similar in our New York Times subcollection (Figure 4a right).

We also analyze which pairs of visual attribute developers commonly use when encoding multiple quantitative data fields using the same mark (Figure $4 \mathrm{~b}$ ). We find that they primarily follow the approach of using the most perceptually effective visual attributes $[11,13]$ for encoding quantitative data - i.e. combinations of $x$-, $y$-position, area, width and height are most prevalent. Again, the pattern is similar for the New York Times subcollection (Figure $4 \mathrm{~b}$ right).

Use of integral/separable attribute pairs: One challenge with multiple encodings is that one encoding may interfere with the perceptual effectiveness of another encoding. Graphical perception researchers call pairs of attributes integral if viewers have difficulty reading one without interference from the other and call them separable otherwise $[14,16]$. To avoid interference attributes that are integral should ideally encode data that is highly correlated while attributes that are separable can encode correlated or uncorrelated data. Figure $4 \mathrm{c}$ shows for each pair of data-encoding attributes, how well the corresponding data fields are 
(C) 2019 IEEE. This is the author's version of the article that has been published in IEEE Transactions on Visualization and Computer Graphics. The final version of this record is available at: 10.1109/TVCG.2019.2934431

Query



15 results tound

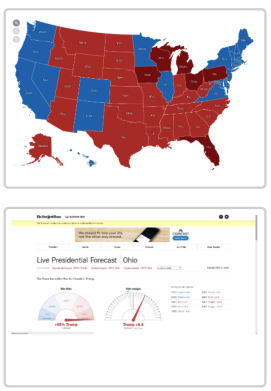

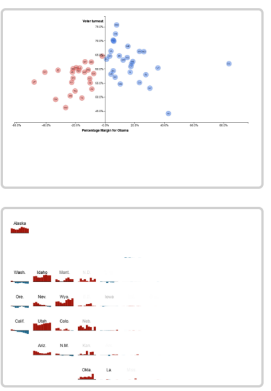
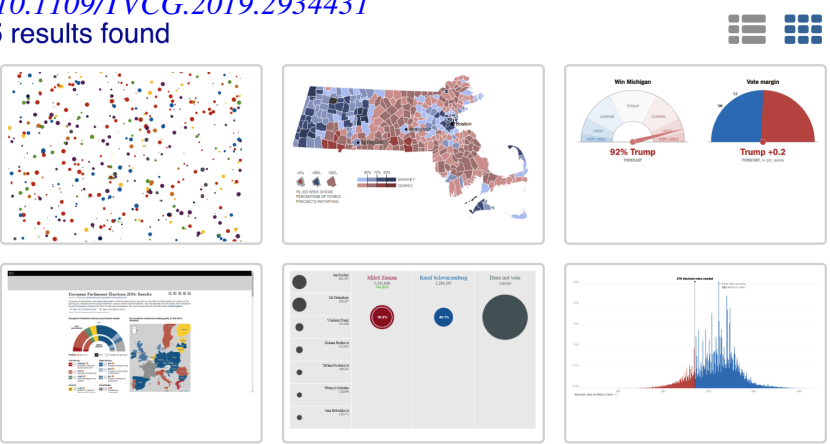

Fig. 2. Query for charts from webpages containing the word "US Election" and that use red and blue as fill-colors on data-encoding marks. The results include a variety of different charts showing the divisions between democrats (usually encoded using blue marks) and republicans (usually encoded using red marks).


10 results found

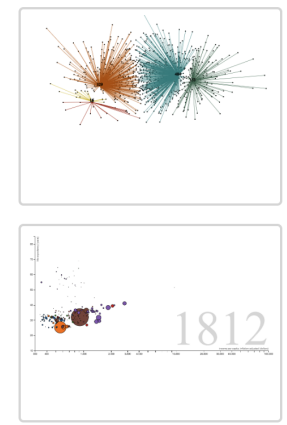

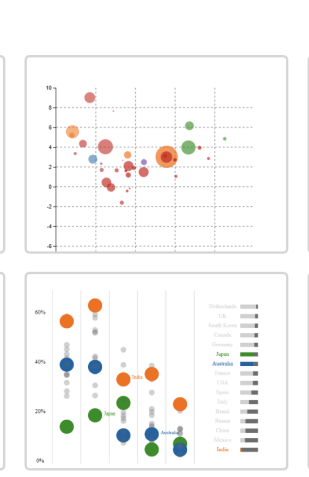

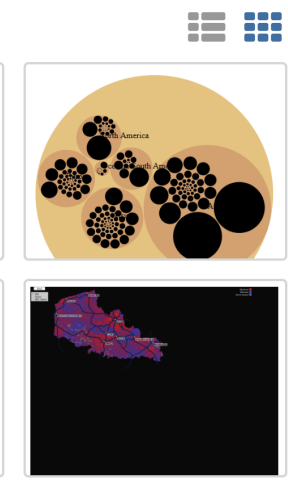

Fig. 3. Query for charts containing a data field with name semantically similar to the word 'population' according to our ' "wordSim' ' similarity operator. Our search engine finds 10 charts related to "population" including several D3 implementation of the GapMinder visualization of Hans Rosling [4]. The data fields these charts do not always include the name "population" but include related terms such as "people" and "human".

correlated. We find that the Pearson correlation coefficients cover the whole range of possible values for the first three pairs of attributes. For many of the pairs lower in the list, the encoded data fields tend to either be uncorrelated (near 0 correlation) or highly correlated (near 1 or sometimes -1) with a few scattered examples in between. Pairs of attributes that are known to be highly integral such as width and height are primarily used to encode highly correlated data fields. The pattern is even stronger for our New York Times subcollection (Figure 4c right). These results suggest that visualization developers, especially at the New York Times, typically follow good practices of using integral pairs of encoding attributes when the data fields are correlated.

Use of fill-color encodings: Visualization developers and researchers have established best practices for using fill-color to encode data. For example, a rule of thumb is to limit the number of colors used to encode nominal data to between six and twelve, so that viewers can perceptually discriminate between them $[13,16]$. Using our search engine we find that among the visualizations that use fill-color to encode nominal data, $58.0 \%$ use six or more different colors while $13.6 \%$ use twelve or more different colors (Figure 5). However, in the New York Times subcollection, we find that there was not a single chart using eight or more different colors to encode nominal data but that $47.9 \%$ use six or more different colors. These results suggest that in practice developers commonly use 6 or more colors for nominal encodings, but avoid using 12 or more colors.

Using more than six colors for encodings nominal data can be especially problematic when marks are small as it is difficult to perceptually discriminate the fill-color of small marks [13]. We used our search engine to find charts encoding nominal data using more than six fillcolors and containing small sized marks. Manually examining these results we find that most are scatterplots with small marks representing the points and indeed it is challenging to discriminate the colors of these points. We expect that this type of access to problematic design examples could help visualization developers better understand such underlying problems.

\section{Extensions to D3 Deconstructor}

In developing our search engine for D3 visualizations we have extended Harper and Agrawala's D3 deconstruction algorithm $[9,10]$ to extract additional structure and style information from an input D3 chart.

\subsection{Extracting angle attributes for arc type marks}

In D3 pie charts, donut charts and radial bar charts are created using marks instantiated with $\mathrm{d} 3 \cdot \operatorname{arc}()$ and encode data based on the internal angle of the arc (or pie slice). Harper and Agrawala's deconstructor does not deconstruct such arc marks and therefore cannot properly extract the encodings for pie charts, donut charts and radial bar charts. We address this problem by directly recovering the internal angle for such marks and then computing whether a data field is mapped to angle.

Consider a pie chart with several slices (or arcs). Internally, D3 uses an SVG path element to construct the geometry of an arc and binds four additional properties to each such path element; startAngle, endAngle, innerRadius and outerRadius. For pie charts, the innerRadius is 0 , whereas for donut charts the innerRadius is set to a positive value. We extract these four properties from each such path element representing an arcs and then compute the angle for the arc as angle $=($ endAngle - startAngle $)$. Finally, as in Harper and Agrawala, we test if there is a linear relationship between the values of every numeric data field and the extracted angles using linear regression. If we find a linear relationship, we consider the the data field to be encoded by angle.

\subsection{Extracting non-data-encoding visual attributes}

Harper and Agrawala's deconstructor does not extract visual attributes for marks and SVG elements that do not encode data. For example, the background color, height and width of the root SVG element are important non-data-encoding visual attributes of the overall visualization. Similarly the typeface, fontStyle, stroke-width, etc. can be non-data-encoding attributes of marks. We extract these additional visual attributes to enable search by non-data-encoding stylistic properties of the charts. To extract this information we traverse the SVG 
(C) 2019 IEEE. This is the author's version of the article that has been published in IEEE Transactions on Visualization and Computer Graphics. The final version of this record is available at: 10.1109/TVCG.2019.2934431

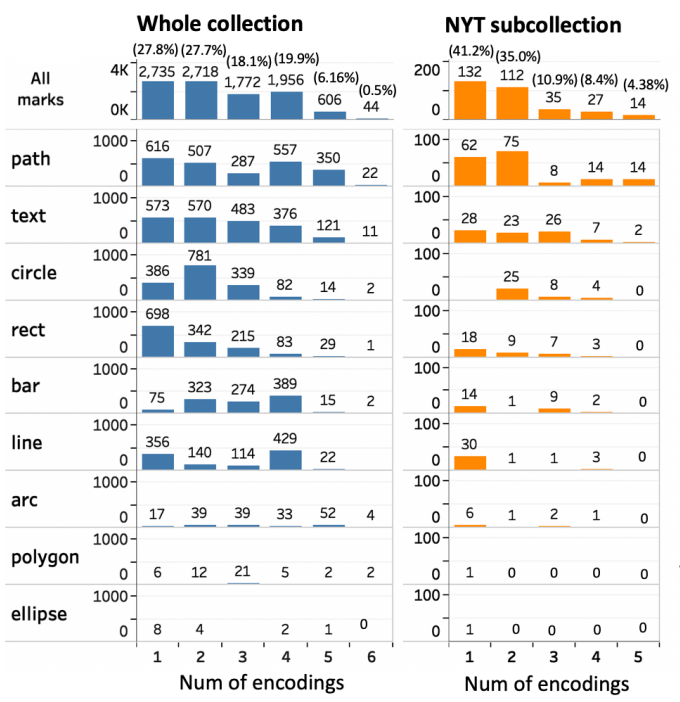

(a) Histogram of number of encodings for each mark type

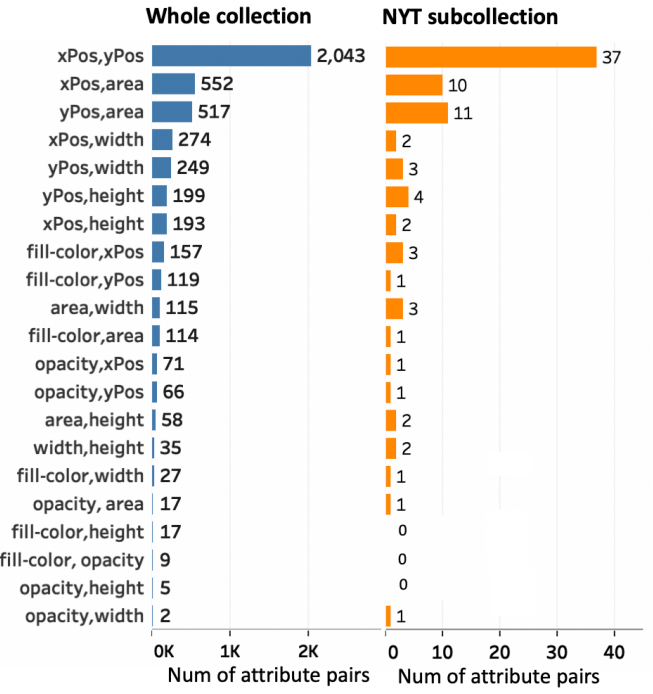

(b) Use of pairs of mark attributes



(c) Pearson correlation

Fig. 4. Histogram of number of encodings for each mark type (a) showing that while $27.8 \%$ of data-encoding marks encodes a single attribute, $72.2 \%$ encode more than one attribute. Across the whole collection the maximum number of encoding attributes for a mark is 6 , whereas for the New York Times subcollection it is 5 . Counts of the numbers of data-encoding attribute pairs for the same mark (b) showing that position, area, height and width are most commonly used together. For each data-encoding attribute pair the correlation coefficients for the underlying data (c) show that pairs of attributes that are integral tend to depict correlated (coefficient is 1 or -1 ) data.

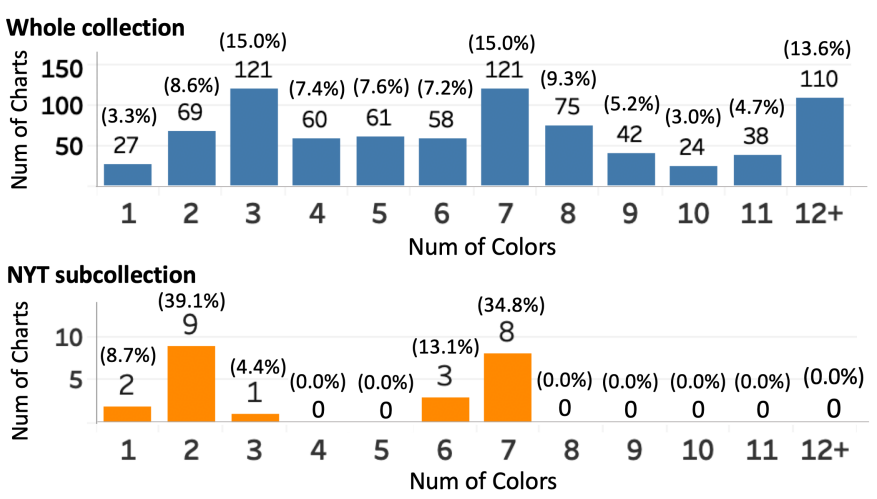

Fig. 5. Histograms of the number of colors used when when fill-color is used to encode nominal data.

subtree of the DOM from the root SVG node for the visualization to the children and look up the non-data-encoding properties of each such node using an SVG parser library [6].

\subsection{Extracting axes and their attributes}

Harper and Agrawala's deconstructor identifies axes by assuming that D3 groups the marks of an axis and stores a axis scale object with the group. Their algorithm checks whether each SVG group node has a scale object and if so all the marks under that group are considered as parts of an axis (e.g. axis line, tick marks, tick labels).

In D3 Versions 4 and 5 (V4 and V5) axes are created and the corresponding scale objects are stored in a different way. For example, in V3 the developer had to use two separate constructors namely d3.svg.axis() and axis.orient () to initiate the axis. In contrast, in later versions, the developer just needs to provide one of the four constructors for each orientation: d3.axisTop(), d3.axisRight (), d3. axisBottom(), d3. axisLeft (). Also, V4 changes how it stores the scale object associated with each axis. With V3, the scale object is accessible through __chart_.. In V4 and V5, the scale object is stored as _-axis instead. We updated our deconstructor so that if it does not find __chart__, it checks if __axis exists and if so it checks the axis orientation ' "orient' '. If the orientation is either top or bottom, it considers the axis to be an $\mathrm{x}$-axis, and otherwise (either left or right) it is considered a y-axis.

Harper and Agrawala's deconstructor also does not identify axis titles which are used to describe the data represented by an axis (e.g. "title": "date" for x-axis and "title": "average price" for y-axis). In order to extract such axis titles we first identify the set of SVG text elements that do not encode data. For each SVG text element that our deconstructor has marked as not encoding data we use a rule-based approach to check whether it represents a title for the $\mathrm{x}$ - or $y$-axis. We compute the proximity of the candidate text element to the axis tick marks and axis lines. Specifically, if the distance between the $\mathrm{y}$-coordinate of the candidate text and y-coordinate of the $\mathrm{x}$-axis tick marks is under a threshold $t$ and the x-coordinate of starting point of the text element is within the boundary of the $\mathrm{x}$-axis line we consider the text to be the $\mathrm{x}$-axis title. We perform the analogous check for proximity the y-axis. We empirically set the value of $t=50$. Any text elements that do not meet either of these two criteria are labeled as 'other text labels' (e.g. chart title, chart annotation text).

\section{USER StUdy: TASKS}

Task 1: You are a visualization developer and you have a dataset containing one quantitative and two categorical data fields. You are interested in creating a chart that shows this dataset. For this purpose, you need to find a set of example charts that would provide ideas for your design and whose D3 cdoe you might reuse or adapt for your purpose. Your task is to find five different charts that are suitable for showing your dataset using the given search interface.

Task 2: You are a visualization developer and you have a dataset containing at least two quantitative and one categorical data fields. You also have over 100 data points in your dataset. You are interested in creating a scatterplot that shows the dataset. For this purpose, you need to find a set of example charts that would provide ideas for your design and whose D3 cdoe you might reuse or adapt for your purpose. Your task is to find five different charts that are suitable for showing your dataset using the given search interface.

\section{REFERENCES}

[1] Big list of D3 examples, 2019. Retrieved March 20, 2019 from http: //christopheviau.com/d3list/.

[2] Blocks, 2019. Retrieved March 20, 2019 from https://bl.ocks.org.

[3] D3.js, 2019. Retrieved March 20, 2019 from https://d3js.org.

[4] Gapminder, 2019. Retrieved March 20, 2019 from https://www. gapminder.org/tools/. 
(C) 2019 IEEE. This is the author's version of the article that has been published in IEEE Transactions on Visualization and Computer Graphics. The final version of this record is available at: 10.1109/TVCG.2019.2934431

[5] New York Times Interactive, 2019. Retrieved March 20, 2019 from http://www.nytimes.com/newsgraphics/2013/12/30/ year-in-interactive-storytelling/index.html.

[6] SVG parser library, 2019. Retrieved March 16, 2019 from https:// github.com/canvg/canvg.

[7] Wall Street Journal, 2019. Retrieved March 20, 2019 from http:// graphics.wsj.com.

[8] Word embedding trained on Googole News, 2019. Retrieved March 17, 2019 from https: //code.google.com/archive/p/word2vec/.

[9] J. Harper and M. Agrawala. Deconstructing and restyling D3 visualizations. In Proc. of UIST, pp. 253-262. ACM, 2014.

[10] J. Harper and M. Agrawala. Converting basic D3 charts into reusable style templates. IEEE TVCG, pp. 1274-1286, 2017.

[11] J. Mackinlay. Automating the design of graphical presentations of relational information. ACM Trans. on Grap. (TOG), 5(2):110-141, 1986.

[12] T. Mikolov, K. Chen, G. Corrado, and J. Dean. Efficient estimation of word representations in vector space. arXiv:1301.3781, 2013.

[13] T. Munzner. Visualization Analysis and Design. CRC Press, 2014.

[14] S. E. Palmer. Vision science: Photons to phenomenology. 1999.

[15] J. Sechler, L. Harrison, and E. M. Peck. Sightline: Building on the web's visualization ecosystem. In Proc. of SIGCHI, pp. 2049-2055. ACM, 2017.

[16] C. Ware. Information visualization: perception for design. Elsevier, 2012. 